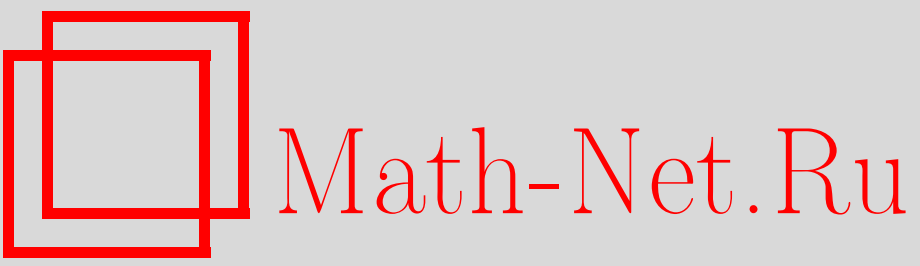

Г. А. Мартынов, Термодинамика и гидродинамика (статистическое обоснование). 1. Постановка задачи, ТМФ, 2002, том 133, номер 1, 121-131

DOI: https://doi.org/10.4213/tmf384

Использование Общероссийского математического портала Math-Net.Ru подразумевает, что вы прочитали и согласны с пользовательским соглашением http: //www.mathnet.ru/rus/agreement

Параметры загрузки:

IP: 35.173 .137 .237

26 апреля 2023 г., 08:37:32 
ТЕОРЕТИЧЕСКАЯ

И МАТЕМАТИЧЕСКАЯ

ФИЗИКА

Том 133, № 1

октябрь, 2002

(C) 2002 г.

\author{
Г. А. Мартынов*
}

\title{
ТЕРМОДИНАМИКА И ГИДРОДИНАМИКА (СТАТИСТИЧЕСКОЕ ОБОСНОВАНИЕ). \\ 1. ПОСТАНОВКА ЗАДАЧИ
}

Проанализированы физические предпосылки статистической механики и показано, что она может быть только локальной. Это означает, что структура вещества и все его макроскопические параметры (за исключением плотности $\rho$, скорости жидкости $\vec{v}$ и температуры $\theta$ ) должны определяться тем, что происходит внутри корреляционной сферы радиуса $R \approx 10^{-7}$ см; значения же $\rho, \vec{v}$ и $\theta$, играющие роль заданных параметров для иерархии ББГКИ, должны находиться путем решения уравнений гидродинамики с учетом макроскопических граничных и начальных условий.

Ключевые слова: иерархия ББГКИ, термодинамический предел, корреляционная сфера, локальный подход.

\section{1. ВВЕДЕНИЕ}

Очевидно, что в основе статистической механики должны лежать уравнения движения классической механики, описывающие движение каждой частицы системы в отдельности. Из них для равновесных систем должны следовать распределение Гиббса и законы термодинамики, а для неравновесных - кинетические уравнения и уравнения гидродинамики. Первый и наиболее важный шаг по пути создания такой обшей теории был сделан Лиувиллем, а затем Боголюбовым, Борном, Грином, Кирквудом и Ивоном (ББГКИ), сумевшими связать уравнения движения классической механики с аппаратом функций распределения. Этим авторам удалось также наметить пути перехода от микроскопического описания вешества в рамках иерархии ББГКИ к макроскопическим уравнениям термодинамики и гидродинамики, однако из-за больших математических трудностей (и, как мне кажется, из-за отсутствия четких физических представлений) довести до конца намеченную программу так и не удалось. Я полагаю, что если бы была ясна физическая картина тех процессов, которые кроются за уравнениями иерархии ББГКИ, не было бы, наверное, многочисленных неудачных попыток построения теории неравновесных явлений на основе только одного уравнения Лиувилля. И что, пожалуй, еше более важно, из-за незавершенности теории не удалось получить каких-то новых,

${ }^{*}$ Институт физической химии РАН, Москва, Россия. E-mail: gamartyn@mtu-net.ru 
неизвестных до тех пор результатов. В конце концов это привело к тому, что теория ББГКИ, весьма популярная в третьей четверти прошлого века, сейчас незаслуженно забыта - не только в учебниках, но и в фундаментальных монографиях по статистической механике о ней даже не упоминают (см., например, [1]-[4]).

Цель данной серии статей - показать, что иерархия ББГКИ должна лежать в основе всей - как равновесной, так и неравновесной - статистической механики и что подход, основанный на иерархии ББГКИ, открывает новые, ранее неизвестные возможности. Я начну с того, что попытаюсь воссоздать физическую картину, лежащую в основе уравнений иерархии. Безусловно, многое из того, о чем будет идти речь ниже, неоднократно упоминалось в литературе и может считаться хорошо известным. Но все эти элементы до сих пор не удавалось собрать в единую конструкцию.

\section{2. ИЕРАРХИЯ ББГКИ}

В дальнейшем всегда будем рассматривать замкнутую изолированную систему $N$ тождественных друг другу частиц массы $m$, занимающую объем $V$ произвольной формы. Будем считать, что система покоится в лабораторной (инерциальной) системе координат. Как известно, в механике состояние такой системы описывается уравнениями Гамильтона, из которых путем тождественных преобразований может быть получено уравнение Лиувилля для глобальной (т.е. характеризующей состояние всей макроскопической системы как единого целого) $N$-частичной функции распределения $G_{(N)}$, зависяшей от времени $t$ и от координат $\vec{r}_{i}$, импульсов $\vec{p}_{i}$ всех $N$ частиц системы. Однако это уравнение не определяет однозначно функцию $G_{(N)}$, так как не налагает никаких ограничений на взаимное расположение частиц внутри объема системы $V$. Так, например, в случае стационарного состояния решением уравнения Лиувилля является произвольная функция $f\left(H_{(N)}\right)$, где $H_{(N)}$ - гамильтониан системы. Определить конкретньй вид $f$, оставаясь в рамках представлений, лежаших в основе уравнения Лиувилля, невозможно. Неоднозначность функции $G_{(N)}$, по всей вероятности, можно устранить только путем перехода от $G_{(N)}$ к $l$-частичным функциям распределения [5]

$$
\begin{aligned}
G_{(l)}\left(\vec{r}_{1}, \ldots, \vec{r}_{l}, \vec{p}_{1}, \ldots, \vec{p}_{l}, t\right)=\frac{1}{V P^{3}} \int_{V} d \vec{r}_{l+1} \times \\
\times \int_{-\infty}^{+\infty} G_{(l+1)}\left(\vec{r}_{1}, \ldots, \vec{r}_{l+1}, \vec{p}_{1}, \ldots, \vec{p}_{l+1}, t\right) d \vec{p}_{l+1}
\end{aligned}
$$

описывающим взаимное расположение элементов группы, состоящей из $l=1,2, \ldots, N$ частиц, т.е. определяющим структуру группы. Здесь $P$ - нормировочный множитель, конкретное значение которого на данном этапе несущественно.

С помошью соотношений (1) можно тождественным образом преобразовать уравнение Лиувилля в иерархию ББГКИ [5]

$$
\frac{\partial G_{(l)}}{\partial t}=\sum_{i=1}^{l}\left\{-\frac{\vec{p}_{i}}{m} \frac{\partial G_{(l)}}{\partial \vec{r}_{i}}+\frac{\partial U_{(l)}}{\partial \vec{r}_{i}} \frac{\partial G_{(l)}}{\partial \vec{p}_{l}}+\frac{N-l}{V P^{3}} \int_{V} d \vec{r}_{l+1} \int_{-\infty}^{+\infty} \frac{\partial \Phi_{i, l+1}}{\partial \vec{r}_{i}} \frac{\partial G_{(l+1)}}{\partial \vec{p}_{i}} d \vec{p}_{l+1}\right\},
$$


где $l=1,2, \ldots, N$, а потенциальная энергия $U_{(l)}$ группы $l$ частиц задается соотношениem

$$
U_{(l)}=\sum_{l=1}^{l} \Phi_{i}+\sum_{1 \leqslant i<j \leqslant l} \Phi_{i j}
$$

Здесь $\Phi_{i} \equiv \Phi_{(i)}\left(\vec{r}_{i}\right)$ - потенциал внешних сил, $\Phi_{i j} \equiv \Phi_{(2)}\left(r_{i j}\right)$ - энергия парного взаимодействия частиц, $r_{i j}=\left|\vec{r}_{i}-\vec{r}_{j}\right|$. Обычно этот потенциал обрашается в нуль на расстояниях $r>R_{\Phi} \approx 10^{-7}$ см. В результате все интегралы в выражении (2) фактически берутся не по всему объему системы $V$, а по объему сферы $V_{R} \approx R_{\Phi}^{3} \approx 10^{-21} \mathrm{~cm}^{3}$. Действительно, сделаем в функциях распределения $G_{(l+1)}=G_{(l+1)}\left(\vec{r}_{1}, \ldots, \vec{r}_{i}, \vec{r}_{l+1}\right)$ замену переменных $\vec{r}_{j}=\vec{r}_{i}+\vec{r}_{i j}$. После этого их можно записать в виде $G_{(l+1)}=$ $G_{(l+1)}\left(\vec{r}_{i} ; \vec{r}_{i 1}, \ldots, \vec{r}_{l, l+1}\right)$ и перейти в $(2)$ от интегрирования по $d \vec{r}_{l+1}$ к интегрированию по разности координат $d \vec{r}_{i, l+1}$. Так как потенциал $\Phi_{i, l+1}=\Phi_{(2)}\left(r_{i, l+1}\right)$ обращается в нуль на расстояниях $r_{i, l+1}>R_{\Phi}$, то очевидно, что вместе с ним обрашается в нуль и все подынтегральное выражение (здесь $r_{i, l+1}=\left|\vec{r}_{i, l+1}\right|$ ).

\section{3. ГРАНИЧНЫЕ УСЛОВИЯ}

Так как уравнения иерархии содержат не сами функции распределения, а их производные, к ним необходимо добавить начальные условия по $t$ и граничные условия по $\vec{r}_{i}, \vec{p}_{i}$. Только после этого можно надеяться, что решение ББГКИ будет однозначно определено (я говорю “можно надеяться" потому, что в настоящее время это утверждение не доказано).

Формулировка начальных и граничных условий эквивалентна выбору тех объектов, которые в дальнейшем предполагается рассматривать. Поэтому значения их должны выбираться из физических соображений. В следуюших статьях будет показано, что теория как равновесных, так и неравновесных систем строится в предположении независимости функций распределения от времени. Поэтому для них вопрос о задании начальных условий отпадает сам собой (в случае метастабильного состояния вещества это, по всей вероятности, не так). Но проблема формулировки граничных условий по $\vec{r}_{i}$ и $\vec{p}_{i}$ остается.

В конечном счете любая физическая теория представляет собой обобщение экспериментальных данных. Поэтому для выбора граничных условий мы должны обратиться к эксперименту, но только не к реальному, а к численному. Из данных, полученных методом молекулярной динамики, следует, что, во-первых, вероятность обнаружения быстрых частиц тем меньше, чем больше их скорость, и во-вторых, корреляция в поведении частиц тем меньше, чем больше расстояние между ними. Напомним, что в молекулярной динамике проводится численное решение уравнений Гамильтона, причем никаких априорных предположений о поведении корреляций на больших расстояниях не делается [6]. Соответственно будем считать, что [5]

$$
G_{(l)} \rightarrow 0 \quad \text { при } \quad \vec{p}_{i} \rightarrow \pm \infty
$$


и

$$
G_{1, \ldots, l} \rightarrow \prod_{i=1}^{l} G_{i} \quad \text { при } \quad \vec{r}_{i} \rightarrow \infty, \quad l \leqslant i \leqslant l,
$$

где нижний индекс без скобки указывает номера частиц, от которых зависит данная функция (последнее условие обычно называется условием ослабления корреляций). Согласно (5) при разлете частиц данной группы на бесконечность всякое взаимодействие меж ду ними исчезает и вероятность их обнаружения оказывается равной произведению вероятностей (независимые события). Тем самым условие ослабления коррелящий налагает определенные ограничения на внутреннюю структуру вешества, т.е. вводит в теорию именно тот элемент, которого не хватало теориям, основанным на уравнении Лиувилля.

Кроме того, поскольку мы трактуем функции распределения $G_{(l)}$ как вероятности соответствуюших событий, на них необходимо наложить условие нормировки

$$
1=\frac{1}{V P^{3}} \int G_{(1)} d \vec{r} d \vec{p}
$$

Соотношения (4)-(6) достаточно тривиальны, и их можно было бы сформулировать, не обрашаясь к эксперименту. Но тот факт, что условия (4)-(6), играюшие роль постулатов, непосредственно следуют из уравнений Гамильтона, еще раз подчеркивает генетическую связь статистической механики с классической механикой.

Следует отметить, что эти постулаты приводят к ряду весьма важных следствий, на которые, как мне кажется, до сих пор не обращали должного внимания. Действительно, из того факта, что при $r>R_{\Phi}$ подынтегральные выражения в иерархии ББГКИ обращаются в нуль, еще не следует, что на этих же расстояниях обращаются в нуль и решения этих уравнений. Однако согласно (5) функция $h_{1, \ldots, l}=G_{1, \ldots, l}-\prod_{i=1}^{l} G_{i}$ может быть отлична от нуля только на малых расстояниях. В следующей статье будет показано, что $h_{(l)} \rightarrow 0$ по экспоненциальному закону. Это убывание является столь резким, что всегда можно ввести представление о радиусе корреляции $R$ и считать, что $h_{(l)}=0$ при $r>R$. Многочисленные расчеты показывают, что в большинстве случаев $R \approx R_{\Phi}$, где $R_{\Phi}-$ расстояние, на котором потенциал взаимодействия $\Phi(r)$ обрашается в нуль (исключением является лишь область вблизи критической точки). Это означает, что при $V>V_{R}$ решение иерархии ББГКИ перестает зависеть от величины $V$. Поэтому при выполнении этого условия величиной $V$ можно распоряжаться по собственному желанию, поскольку значения функций распределения уже не зависят от $V$. А так как функции распределения определяют все макроскопические параметры вешества [7], то при $V>V_{R}$ последние также не зависят от конкретного значения $V$. В частности, всегда можно положить $V=\infty$, ничего при этом не меняя ни в исходных уравнениях, ни в полученных с их помошью результатах.

Пусть объем системы $V \approx L^{3}$, где $L$ - характерный макроскопический размер. Очевидно, что выполнение неравенства

$$
R \ll L
$$


гарантирует независимость интегралов в (1) и (2) от размеров и формы системы. А так как радиус корреляции $R$ в отличие от $V$ нельзя менять произвольно, то неравенство (7) фактически налагает ограничения на размер $L$ исследуемой системы: он всегда должен быть много больше радиуса корреляционной сферы. По сушеству, это неравенство является определением понятия "макроскопическое тело", а объем корреляционной сферы $V_{R}$ играет роль того физически бесконечно малого объема $\delta V$, для которого в макроскопической теории процессов переноса составляются уравнения баланса массы, импульса и энергии.

Еше одно замечание. Согласно условию ослабления корреляший (5) функция $h_{1, \ldots, l}=$ $G_{1, \ldots, l}-\prod_{i=1}^{l} G_{i}$ отлична от нуля только при $r<R$. Поскольку значения $h_{(l)}$ определяются уравнениями иерархии ББГКИ, то внутри корреляционной сферы распределение плотности является детерминированным (предполагается, что уравнения иерархии имеют единственное решение). В то же время вне корреляционной сферы распределение плотности уже не подчиняется законам, диктуемым уравнениями иерархии - мгновенные значения параметров вещества в области $r>R$ могут быть найдены только путем решения исходных уравнений Гамильтона. Но последние, как это следует из данных молекулярной динамики, порождают хаос. Поэтому состояние вешества вне корреляционной сферы является случайным, недетерминированным. В результате рассматриваемая нами макроскопическая система распадается на две части: микроскопически малую корреляционную сферу и окружаюший ее термостат бесконечных размеров. При этом движение частиш внутри корреляционной сферы является детерминированным, а в термостате - хаотическим (случайным), что и отличает их друг от друга. Отсюда следует, что условие ослабления корреляций, которое мы принимаем в качестве граничного условия, по сушеству, эквивалентно гипотезе молекулярного хаоса, лежашей в основе теории Больцмана.

И последнее. Условие ослабления корреляций налагает ограничения на все функции распределения, кроме одночастичной функции $G_{(1)}(\vec{r}, \vec{p}, t)$. Ее значения определяются не только с помошью иерархии ББГКИ, но и с помошью соотношений, связываюших $G_{(1)}(\vec{r}, \vec{p}, t)$ с макроскопическими параметрами системы. Таких параметров три:

1) плотность числа частиц

$$
\rho(\vec{r}, t)=\rho_{0} \int_{V} G_{(1)}(\vec{r}, \vec{p}, t) \frac{d \vec{p}}{P^{3}}
$$

$2)$ скорость макроскопического течения жидкости $\vec{v}(\vec{r}, t)$, входяшая в формулу

$$
m \rho(\vec{r}, t) \vec{v}(\vec{r}, t)=\rho_{0} \int_{-\infty}^{+\infty} \vec{p} G_{(1)}(\vec{r}, \vec{p}, t) \frac{d \vec{p}}{P^{3}}
$$

3) температура $\theta(\vec{r}, t)$, определяюшая среднее значение кинетической энергии частиц

$$
\frac{3}{2} \rho(\vec{r}, t) \theta(\vec{r}, t)=\rho_{0} \int_{-\infty}^{+\infty} \frac{(\vec{p}-m \vec{v}(r, t))^{2}}{2 m} G_{(1)}(\vec{r}, \vec{p}, t) \frac{d \vec{p}}{P^{3}},
$$


где $\rho_{0}=N / V$. С помошью этих соотношений устанавливается связь функций распределения с макроскопическим состоянием системы. При этом стояшие слева макроскопические параметры теории $\rho(\vec{r}, t), \vec{v}(\vec{r}, t), \theta(\vec{r}, t)$ должны считаться заданными, а функция $G_{(1)}(\vec{r}, \vec{p}, t)$ должна подбираться таким образом, чтобы удовлетворялись соотношения (8)-(10). Как известно, гидродинамические переменные $\rho(\vec{r}, t), \vec{v}(\vec{r}, t), \theta(\vec{r}, t)$ в макроскопической теории определяются уравнениями гидродинамики, а также граничными и начальными условиями к ним ${ }^{1}$. Они являются тем звеном, которое устанавливает связь между микро- и макромиром. Подчеркнем, что эта связь не вводится нами "by hand", а является следствием иерархии ББГКИ.

Итак, статистическая механика предполагает следуюшее "двухэтажное” описание системы:

а) на микроуровне - локальное описание состояния вещества внутри корреляционной сферы с помощью уравнений иерархии ББГКИ;

б) на макроуровне - глобальное описание состояния всей макроскопической системы с помошью макроскопических уравнений гидродинамики и макроскопических начальных и граничных условий к ним.

В то же время исходные уравнения Гамильтона определяют координаты каждой частицы вешества в отдельности и тем самым задают как микро-, так и макросостояние данного тела. Поэтому формулировка статистической механики может считаться законченной только в том случае, когда мы из иерархии ББГКИ (т.е. фактически из уравнений Гамильтона) получим не только распределение Гиббса, но и уравнения гидродинамики, так как иначе "этажи” теории не могут быть соединены в единую конструкцию.

\section{4. ТЕРМОДИНАМИЧЕСКИЙ ПРЕДЕЛ}

Соотношения (1) и (2) представляют собой две разные (и, возможно, несовместимые друг с другом $\left.{ }^{2}\right)$ системы уравнений для определения одних и тех же функций распределения $G_{(l)}$. Очевидно, что подобная двойственность противоречит требованию однозначности, которое предъявляется к любой физической теории. Для ее устранения необходим переход к термодинамическому пределу.

Чтобы условие ослабления корреляций выполнялось автоматически, положим

$$
G_{i}=e^{\omega_{i}}, \quad G_{1, \ldots, l}=e^{\Omega_{1, \ldots, l}} \prod_{i=1}^{l} G_{i}, \quad \Omega_{1} \equiv 0,
$$

где функции $\omega_{i}, \Omega_{1}, \ldots, l$ заданы уже на всей действительной оси (по определению все $\left.G_{(l)} \geqslant 0\right)$. Подчеркнем, что никаких дополнительных ограничений на вид функций $G_{(l)}$ соотношения (11) не налагают.

\footnotetext{
1) В равновесии уравнения гидродинамики вырождаются в тождества $\rho, \theta=$ const, $\vec{v}=0$, где $\rho, \theta$ могут иметь произвольные значения. Но и в этом случае гидродинамические параметры должны вводиться в теорию с помощью соотношений (8) и (10).

2) Подобная возможность следует хотя бы из того, что уравнения (1) не содержат потенциалов взаимодействия $\Phi_{i j}$, тогда как в иерархии ББГКИ (2) эти потенциалы играют решаюшую роль.
} 
Из условия ослабления корреляции следует, что все функции

$$
\Omega_{1, \ldots, l} \rightarrow 0 \quad \text { при } \quad \vec{r}_{1}, \ldots, \vec{r}_{l} \rightarrow \infty .
$$

В этих обозначениях иерархия ББГКИ записывается в виде

$$
\begin{gathered}
\frac{\partial \omega_{1}}{\partial t}=-\frac{\vec{p}_{1}}{m} \frac{\partial \omega_{1}}{\partial \vec{r}_{1}}-\vec{F}_{1} \frac{\partial \omega_{1}}{\partial \vec{p}_{1}}-\frac{\partial \vec{F}_{1}}{\partial \vec{p}_{1}}, \\
\frac{\partial \Omega_{(l)}}{\partial t}=-\sum_{i=1}^{l}\left\{\left(\vec{F}_{(l)}-\vec{F}_{i}\right) \frac{\partial \omega_{i}}{\partial \vec{p}_{i}}+\frac{\vec{p}_{i}}{m} \frac{\partial \Omega_{(l)}}{\partial \vec{r}_{i}}+\vec{F}_{(l)} \frac{\partial \Omega_{(l)}}{\partial \vec{p}_{i}}+\frac{\partial\left(\vec{F}_{(l)}-\vec{F}_{i}\right.}{\partial \vec{p}_{i}}\right\},
\end{gathered}
$$

где силы, действуюшие на частишы, заданы соотношениями

$$
\begin{aligned}
\vec{F}_{1, \ldots, l}^{(i)}= & -\frac{\partial U_{1, \ldots, l}}{\partial \vec{r}_{i}}-\frac{N-l}{V} \int_{V} d \vec{r}_{l+1} \times \\
& \times \int_{-\infty}^{+\infty} \frac{\partial \Phi_{i, l+1}}{\partial \vec{r}_{i}} \exp \left[\omega_{l+1}+\left(\Omega_{1, \ldots, l+1}-\Omega_{1, \ldots, l}\right)\right] \frac{d \vec{p}_{l+1}}{P^{3}} .
\end{aligned}
$$

В (14) первый член в правой части определяет силу, действующую на частицу $i$ со стороны остальных $l-1$ частиц данной группы, а интегральный член - среднюю силу, действуюшую на ту же частицу со стороны остальных $N-l$ частиц системы, по координатам которых производится усреднение (предполагается, что координаты всех $l$ частиц данной группы фиксированы).

Наряду с функциями $\Omega_{(l)}$ можно также ввести термические потенциалы $\omega_{1, \ldots, l} \equiv$ $\omega_{(l)}\left(\vec{r}_{1}, \ldots, \vec{r}_{l} ; \vec{p}_{1}, \ldots, \vec{p}_{l} ; t\right)$, связанные с $\Omega_{(l)}$ соотношениями

$$
\begin{aligned}
\Omega_{12} & =\omega_{12}, \\
\Omega_{123} & =\left(\omega_{12}+\omega_{13}+\omega_{23}\right)+\omega_{123} \text { и т.д. }
\end{aligned}
$$

Связь между функциями $\Omega_{(l)}, \omega_{(l)}$ является взаимно-однозначной: зная $\Omega_{(l)}$, всегда можно найти $\omega_{(l)}$, так как

$$
\begin{aligned}
\omega_{12} & =\Omega_{12}, \\
\omega_{123} & =\Omega_{123}-\left(\Omega_{12}+\Omega_{13}+\Omega_{23}\right) \quad \text { и т.д. }
\end{aligned}
$$

Подставляя эти выражения в формулу $(1)$, определяющую функции распределения $G_{(l)}$, получим

$$
1=\frac{1}{V P^{3}} \int G_{2} e^{\omega_{12}} d \vec{r}_{2} d \vec{p}_{2}, \quad 1=\frac{1}{V P^{3}} \int G_{3} e^{\omega_{13}+\omega_{23}+\omega_{123}} d \vec{r}_{3} d \vec{p}_{3}
$$

и т.д. С помошью условия нормировки (6) этим соотношениям можно придать более удобный вид. Добавляя и вычитая из правой части единицу и учитывая условие нормировки (6), преобразуем (17) в бесконечную систему равенств

$$
\begin{aligned}
& 1=1+\frac{1}{V P^{3}} \int_{-\infty}^{+\infty} d \vec{p}_{2} \int_{V} G_{2}\left(e^{\omega_{12}}-1\right) d \vec{r}_{2}, \\
& 1=1+\frac{1}{V P^{3}} \int_{-\infty}^{+\infty} d \vec{p}_{3} \int_{V} G_{3}\left(e^{\omega_{13}+\omega_{23}+\omega_{123}}-1\right) d \vec{r}_{3} \quad \text { и т.д., }
\end{aligned}
$$


из которой следует, что интегральные члены в (18) всегда должны равняться нулю.

Предположим сначала, что объем системы $V$ конечен. В этом случае равенства (18) преврашаются в систему интегральных уравнений для определения неизвестных функций $\omega_{(l)}$,

$$
\begin{aligned}
I_{(1)}\left(\vec{r}_{1}, \vec{p}_{1}, t\right) & =\int G_{2}\left(e^{\omega_{12}}-1\right) d \vec{r}_{2} d \vec{p}_{2}=0 \\
I_{(2)}\left(\vec{r}_{1}, \vec{r}_{2}, \vec{p}_{1}, \vec{p}_{2}, t\right) & =\int G_{3}\left(e^{\omega_{13}+\omega_{23}+\omega_{123}}-1\right) d \vec{r}_{3} d \vec{p}_{3}=0 \text { и т.д. }
\end{aligned}
$$

Но одни и те же функции $\omega_{(l)}$ не могут одновременно быть решением сразу двух различных систем уравнений - уравнений (19) и уравнений иерархии ББГКИ (2). Поэтому данньй вариант должен быть отброшен. Остается только предположить, что в (18) объем $V$ равен бесконечности. В этом случае проблема совместимости уравнений (1) и (2) снимается, так как уравнения (1) (или, что то же, уравнения (18)) вырождаются в тождества типа $1=1$, вообще не зависяшие от вида функций распределения. Однако для того, чтобы в пределе $V \rightarrow \infty$ интегральные члены в (18) исчезали, необходимо потребовать, чтобы в этом пределе интегралы по объему от функций $\omega_{(l)}$ оставались конечными. Последнее возможно лишш при условии, что

$$
\omega_{(l)} \rightarrow 0 \text { при } \vec{r}_{i} \rightarrow \infty, \quad 1 \leqslant i \leqslant l,
$$

или, переходя к функциям $\Omega_{(l)}$,

$$
\Omega_{1, \ldots, i, \ldots, l} \rightarrow \Omega_{1, \ldots, i-1, i+1, \ldots, l}=\Omega_{(l)} \quad \text { при } \quad \vec{r}_{i} \rightarrow \infty .
$$

Легко сообразить, что в том случае, когда на бесконечность разлетаются сразу все частищы данной группы, условия (21) преврашаются в условия ослабления корреляций. Поэтому отказ от последних автоматически означал бы, что мы работаем с системой конечных размеров, для которой условие однозначности теории не выполняется.

Заметим теперь, что поскольку перед каждым интегралом в (2) стоит множитель $N(1-l / N) / V$, то в пределе $V=\infty$ интегральные члены вообще должны исчезнуть из иерархии ББГКИ, что, конечно, недопустимо, так как они описьвают коллективное воздействие $N-l$ частиц системы на выделенную нами группу $l$ частиц. Сохранить интегральные члены можно, перейдя к термодинамическому пределу

$$
N, V \rightarrow \infty, \quad \rho_{0}=\frac{N}{V}=\mathrm{const}
$$

(при более точной формулировке термодинамического предела необходимо также учитьвать неизменность кинетической энергии частиц, т.е. их температуры; см. следующую статью). В этом пределе иерархия ББГКИ принимает вид

$$
\begin{aligned}
\frac{\partial G_{(l)}}{\partial t}= & \sum_{i=1}^{l}\left\{-\frac{\vec{p}_{i}}{m} \frac{\partial G_{(l)}}{\partial \vec{r}_{i}}+\frac{\partial U_{(l)}}{\partial \vec{r}_{i}} \frac{\partial G_{(l)}}{\partial \vec{p}_{l}}+\right. \\
& \left.+\rho_{0} \int_{V} d \vec{r}_{l+1} \int_{-\infty}^{+\infty} \frac{\partial \Phi_{i, l+1}}{\partial \vec{r}_{i}} \frac{\partial G_{(l+1)}}{\partial \vec{p}_{i}} \frac{d \vec{p}_{l+1}}{P^{3}}\right\}, \quad l=1,2, \ldots, \infty
\end{aligned}
$$


По всей вероятности, до перехода к термодинамическому пределу уравнения (2) и (19) имеют разные решения; после перехода эта двойственность исчезает. Поэтому условие (22) является необходимым (хотя, возможно, и не достаточным) шагом на пути построения физической теории, удовлетворяюшей требованию однозначности. Этот вывод находится в полном соответствии с теоремой Ван Хова, согласно которой в частном случае равновесных систем микроканонический, канонический и большой канонический ансамбли приводят к одним и тем же результатам только в термодинамическом пределе [7]. По существу, приведенные здесь соображения являются обобщением этой теоремы на случай неравновесных систем.

\section{5. СЛЕДСТВИЯ ТЕРМОДИНАМИЧЕСКОГО ПРЕДЕЛА}

Переход к термодинамическому пределу сушественно меняет наши представления о статистической механике.

Во-первых, теряется связь между исходным уравнением Лиувилля и иерархией ББГКИ, поскольку определение $l$-частичных функций распределения (1), с помощью которого проводилось преобразование уравнения Лиувилля в иерархию ББГКИ, в этом пределе вырождается в тождество $1=1$. В результате уравнение Лиувилля вообще выпадает из теории.

Во-вторых, статистическая механика из глобальной теории, описывающей состояние сразу всей макроскопической системы, преврашается в локальную теорию, описывающую состояние вешества только внутри корреляционной сферы, проведенной вокруг данной точки.

В-третьих, при конечных значениях локальных параметров $s, e$ и т.д. и $N \rightarrow \infty$ все глобальные характеристики системы (энтропия $S_{(N)}=N s$, глобальная энергия $E_{(N)}=$ $N e$ и т.д.) после перехода к термодинамическому пределу обрашаются в бесконечность,

$$
S_{(N)}=N s \rightarrow \infty, \quad E_{(N)}=N e \rightarrow \infty \text { при } N \rightarrow \infty,
$$

и теряют физический смысл. В результате после перехода $\kappa$ термодинамическому пределу статистическая механика из глобальной превращается в последовательно локальную теорию (последовательную постольку, поскольку в этом пределе из нее исключаются все глобальные макроскопические параметры $S_{(N)}, E_{(N)}$ и т.д., а также глобальная $N$-частичная функция распределения $\left.G_{(N)}\right)$.

В-четвертых, только в термодинамическом пределе уравнения иерархии ББГКИ перестают зависеть от формы и размеров сосуда, вмешаюшего систему. Благодаря этому становится возможным описание в рамках статистической механики газов и жидкостей, свойства которых не зависят от формы и размеров сосуда.

В-пятых, условия ослабления корреляций, согласно которым $\omega_{(l)} \rightarrow 0$ при $r \rightarrow \infty$, оказываются следствием условия однозначности теории.

И наконец, последнее. Предположим, что при плотной упаковке в корреляционную сферу может войти $\nu$ частиц. В этом случае любой термический потенциал $\omega_{(l)} \mathrm{c} l>\nu$ 
обратится в нуль, потому что частицы с номерами $\nu<i \leqslant l$ будут находиться вне корреляционной сферы. Поэтому должны выполняться условия

$$
\max \left|\omega_{(l)}\right| \rightarrow 0 \text { при } l \rightarrow \infty
$$

Эти соотношения обеспечивают сходимость иерархии ББГКИ [8].

\section{6. ЗАКЛЮЧЕНИЕ}

Как уже отмечалось, в основе любой последовательной статистической теории должны лежать уравнения движения классической механики, описьвающие движение всех $N$ частиц системы. По всей вероятности, единственно возможный путь от уравнений движения к уравнениям статистической механики образует цепочку: уравнения Гамильтона $\Rightarrow$ уравнение Лиувилля $\Rightarrow$ иерархия ББГКИ; другого пути сейчас мы не знаем. Конечным звеном этой цепочки является иерархия ББГКИ, образуюшая тот фундамент, на котором стоит вся остальная статистическая механика. Решения уравнений иерархии будут рассмотрены в следуюших статьях; здесь же я бы хотел подчеркнуть, что поскольку иерархия ББГКИ была получена из уравнений Гамильтона путем тождественных преобразований, она является точным и однозначным следствием уравнений движения классической механики.

Что же показал анализ исходных положений теории? Уравнения Гамильтона неустойчивы по отношению к малейшим ошибкам в задании начальных условий [8]. Поэтому мы должны отказаться от слежения за траекториями частиц и перейти к вероятностному описанию системы. Первый шаг на этом пути - уравнение Лиувилля, определяюшее глобальную (т.е. описываюшую сразу все частицы системы) функцию распределения $G_{(N)}$. Но уравнение Лиувилля не налагает никаких ограничений на взаимное расположение частиц внутри системы и как следствие не определяет однозначно искомую функцию. Этот недостаток может быть устранен только путем перехода от уравнения Лиувилля для $N$-частичной функции распределения к иерархии ББГКИ для $l$-частичных функций распределения $G_{(l)}$, где $l=1,2, \ldots, N$. Введенные таким образом функции $G_{(l)}$ определяют взаимное расположение частиц внутри групп частиц, каждая из которых состоит из $l$ элементов, т.е. определяют структуру данной групшы. По всей вероятности, подобное описание системы является наиболее детальным из всех возможных.

Но недостаточно перейти к математическому аппарату, который в принципе может дать наиболее подробное описание системы; необходимо еше выбрать тот класс динамических систем, который мы собираемся описывать с помошью этого аппарата. В механике подобный выбор всегда производится путем задания начальных и граничных условий к уравнениям движения. То же самое должно быть сделано и в статистической механике. В ней рассматриваются только такие системы, в которых корреляции в поведении отдельных частиц быстро затухают с увеличением расстояния между ними (условие ослабления корреляций). Класс таких систем чрезвычайно широк - фактически в него попадают все окружаюшие нас макроскопические тела. 
Казалось бы, в выборе граничных условий всегда имеется определенный произвол. Но, как мы видели, одни и те же функции распределения $G_{(l)}$, вообще говоря, определяются сразу двумя различными системами уравнений. Эта двойственность исчезает только после перехода к термодинамическому пределу, в результате которого одна из двух систем вырождается в тождества $1=1$. В свою очередь, переход к термодинамическому пределу возможен, только если граничные условия задаются в виде условия ослабления корреляций. В результате всякий произвол в формулировке основных положений теории исчезает: мы обязаны в качестве граничного условия выбрать условие ослабления корреляций, ибо в противном теория перестает быть однозначной. Этот вывод находится в полном соответствии с известной теоремой, доказанной еше Гиббсом, которая утверждает, что распределение Гиббса дает однозначное описание равновесных систем только в термодинамическом пределе [9].

Из условия ослабления корреляций следует, что статистическая механика должна быть локальной. Это означает, что на микроуровне структура вешества и все его макроскопические параметры за исключением гидродинамических переменных должны определяться тем, что происходит внутри коррелящионной сферы радиуса $R \approx 10^{-7}$ см; гидродинамические же переменные, играюшие роль заданных параметров для иерархии ББГКИ, должны находиться путем решения уравнений гидродинамики с учетом граничных и начальных условий к ним. Утверждение, согласно которому в основе всей - как равновесной, так и неравновесной - статистической механики должен лежать локальный подход, является главным результатом данной статьи. Казалось бы, этот вывод находится в явном противоречии с сушествуюшей теорией равновесных систем, в основе которой лежит глобальное распределение Гиббса $G_{(N)}$, описывающее состояние сразу всей системы. В следуюшей статье этот вопрос будет рассмотрен достаточно подробно.

\section{Список литературы}

[1] D. Chowdhury, D. Staufer. Principles of Equilibrium Statistical Mechanics. Weinheim: Wiley-VCH, 2000.

[2] J. Walecka. Fundamentals of Statistical Mechanics. London: World Scientific, 2000.

[3] J. Keizer. Statistical Thermodynamics of Nonequilibrium Processes. New York, Heidelberg, London, Paris, Tokyo: Springer, 1987.

[4] T. Guenault. Statistical Physics. London: Chapman \& Hall, 1995.

[5] Н. Н. Боголюбов. Проблемы динамической теории в статистической физике. Избранные труды. Киев: Наукова думка, 1970.

[6] Г. Э. Норман, В. В. Стегайлов. ЖЭТФ. 2001. Т. 119. № 5. С. 1011.

[7] К. Хуанг. Статистическая механика. М.: Мир, 1966.

[8] Г. А. Мартынов. УФН. 1996. Т. 166. № 10. С. 1105.

[9] Дж. В. Гиббс. Основные принципы статистической механики. М.: ОГИЗ, 1946.

Поступила в редакцию 26.XI.2001 г., после доработки 19.II.2002 г. 